\title{
Acercamiento de los Procesos Alveolares Mediante Ortopedia Prequirúrgica en Pacientes con Labio y Paladar Fisurado
}

\author{
Approaching of the Alveolar Processes Using Presurgical \\ Orthopedics in Patients with Cleft Lip and Palate
}

Jorge Fuentes; ; Mónica Silva*; Mario Cantín ${ }^{*, *, * * *}$ \& Stefano Llermaly*

FUENTES, J; SILVA, M.; CANTÍN, M. \& LLERMALY, S. Acercamiento de los procesos alveolares mediante ortopedia prequirúrgica en pacientes con labio y paladar fisurado. Int. J. Odontostomat., 8(1):119-124, 2014.

RESUMEN: Las fisuras orales son un grupo heterogéneo de malformaciones congénitas de origen multifactorial. La fisura labio-palatina es una de las más frecuentes, producto de la alteración en la fusión de los procesos labiales mediales, laterales y palatinos durante el desarrollo embrionario, y Chile presenta una prevalencia mayor a la mundial. Su tratamiento contempla diversos procedimientos como la técnica prequirúrgica. El objetivo fue evaluar si el tratamiento prequirúrgico prepara a los pacientes con labio y/o paladar fisurado para la cirugía primaria. Se realizó un estudio retrospectivo, que incluyo a 100 pacientes de ambos sexos con fisura de labio y/o paladar atendidos bajo tratamiento de ortopedia prequirúrgica en el Consultorio Miraflores, Temuco, entre los años 2003 y 2012. Las fisuras fueron categorizadas según la clasificación de Kernahan. El $46 \%$ de los tratamientos logró un acercamiento adecuado para la cirugía y $20 \%$ sólo requirió manejo de tejidos blandos. Un $8 \%$ no logró un acercamiento adecuado. Veinticuatro porciento de los pacientes abandono el tratamiento. No se observaron diferencias entre la edad de inicio de tratamiento vs. acercamiento de los procesos alveolares. El manejo de tejidos blandos tuvo una correlación significativa con el tiempo total de tratamiento y el acercamiento de los procesos alveolares. Determinar la cantidad de pacientes que logran un acercamiento adecuado de los procesos alveolares mediante la técnica prequirúrgica, permite al clínico dimensionar el efecto del tratamiento y encontrar problemas que se puden estar presentando e impiden un mejor resultado.

PALABRAS CLAVE: fisuras labio-palatina, etapa prequirurgica, etapa de Grayson.

\section{INTRODUCCIÓN}

Las fisuras orales son un grupo heterogéneo de malformaciones congénitas conocidas de origen multifactorial, en las cuales los factores genéticos y ambientales contribuyen a su etiología.

La contribución genética a la causa de las fisuras es entre un 20 a un $50 \%$, el resto es atribuible a factores ambientales o interacciones genético-ambientales (Bermudez et al., 2009). Para Nazer (2011), alrededor de un $20-25 \%$ de los casos tiene algún factor genético presente, otro $20-25 \%$ tiene antecedentes de factores ambientales y en el resto, no hay causa precisa; además, la etiopatogenia de las fisuras de labio y paladar, y las fisuras aisladas de paladar pareciera ser genéticamente distinta. Según Ford et al. (2010), en los factores genéticos participan múltiples genes, incluyendo homeobox MSX1, el factor de crecimiento TGFb3, el receptor del ácido retinoico RARA y el factor de transcripción ARNT2 entre otros.

En cuanto a los factores ambientales involucrados participarían la vitamina A, colchicina y glucocorticoides. Para Rossell (2009), dentro de los factores ambientales podemos tener i) Infecciones: Virus Influenza A2 y Toxoplasma gondii, ii) Drogas: Fenobarbital, Difenilhidantoina, Trimetadiona, Corticoesteroides, diazepam, Tabaco y Alcohol, y iii) Déficit nutricional: Deficiencias de ácido fólico. En re-

* Departamento de Odontología Integral Adultos, Facultad de Odontología, Universidad de La Frontera, Temuco, Chile.

* Programa de Doctorado en Ciencias Morfológicas, Facultad de Medicina, Universidad de La Frontera, Temuco, Chile.

${ }^{* * *}$ Centro de Investigación en Ciencias Biomédicas, Universidad Autónoma de Chile, Temuco, Chile. 
lación al tabaco, Wyszynski et al. (1997), sugieren una pequeña pero significativa asociación entre las madres fumadoras durante el primer trimestre de gestación y el aumento de riesgo de fisura de labio y paladar. Según Little et al. (2004) las mujeres tienen un $30 \%$ más riesgo de tener un hijo con paladar y/o labio fisurado y un $20 \%$ más de riesgo de tener uno con paladar fisurado si estas fuman durante el embarazo. Concluyen que las pruebas de la existencia de relación entre tabaquismo materno y hendiduras orofaciales son lo bastante sólidas para justificar su uso en las campañas anti-tabáquicas. Por otra partes, un estudio de meta análisis sugiere que la exposición materna a pesticidas está asociada a un modesto, pero significativo riesgo de producir fisuras orales. Sin embargo, el riesgo asociado a la ocupación paternal o su exposición residencial a pesticidas fue encontrado elevado pero no significativo (Romitti et al., 2007).

Otro factor a considerar es la edad de los padres. Según Bille et al. (2005), la edad paterna de 40 o más años tiene un $58 \%$ más probabilidades de tener un hijo con fisuras orales que el grupo de padres entre 20 a 39 años. De igual manera, madres de 40 o más años tienen 1,56 veces más riesgo de tener un hijo con fisura de labio y paladar, comparado con madres en edades entre 20 y 29 años. Por tanto, mayor edad paternal y maternal se asocia un mayor riesgo de fisura de labio y/o paladar, pero una edad mayor paternal y no maternal sólo se asocia a un mayor riesgo de fisura de paladar. Aún no existe evidencia de asociación entre una edad temprana, materna y paterna, con la ocurrencia de fisuras orales.

Dada la enorme variabilidad que pueden presentar las fisuras labiopalatinas, muchos autores a través del tiempo han propuesto diferentes sistemas de clasificación. El esquema más conocido y difundido, por su simplicidad y lectura (Nazer), se basa en la "Y" de Kernahan, complementada con el compromiso de nariz y la amplitud de la fisura alveolar en el recién nacido. La clasificación de las fisuras se realiza de acuerdo a las estructuras comprometidas: labio, encía, paladar óseo y velo del paladar; además si es unilateral completa $(3 / 3)$, incompleta $(2 / 3,1 / 3)$ o micro forma (forma frustra o cicatrizal). Igual definición aplica a las fisuras bilaterales, las cuales pueden ser simétricas ( $3 / 3$ a ambos lados) o asimétricas. Las fisuras del paladar pueden ser completas incluyendo el alveolo, el paladar secundario (posterior al foramen incisivo), velo del paladar y submucosas del velo del paladar (sin unión muscular).
El tratamiento ortopédico prequirúrgico de las fisuras de labio y paladar, ha sido utilizado por muchos años. La escuela moderna de la ortopedia prequirúrgica en labio y paladar fisurado, fue iniciada por McNeil en 1959, quien uso una serie de placas para moldear activamente los segmentos alveolares en la posición deseada. En 1975 Georgide \& Latham introdujeron un aparato activo retenido por pines para, simultáneamenteretraer la premaxila y expandir los segmentos posteriores en un periodo de varios días. Hotz en 1987 describió el uso de una placa ortopédica pasiva para alinear los segmentos fisurados. En 1993 Grayson describió una nueva técnica para moldear el alveolo, labio y nariz en infantes nacidos con labio y paladar fisurado. El modelador nasoalveolar consiste de modelador intraoral con antenas nasales para moldear el reborde alveolar y el cartílago nasal al mismo tiempo (Grayson \& Shetye, 2009).

En la Actualidad existen dos corrientes filosóficas para el tratamiento de pacientes con fisura labiopalatina. Una utiliza solamente la cirugía para la corrección de la deformidad, mientras que la otra corriente incluye, además de la cirugía, una corrección prequirúrgica de los segmentos fisurados. En chile, la Guía clínica de Fisura Labiopalatina del MINSAL (Ministerior de Salud, 2009) recomienda su utilización.

El objetivo de este estudio fue determinar grado de avance de los procesos alveolares logrado en los pacientes con fisura de labio y/o paladar bajo tratamiento de ortopedia prequirúrgica, atendidos en la región de la Araucanía entre los años 2003 y 2012, así como su relación con la cantidad de controles y tiempo de tratamiento.

\section{MATERIAL Y MÉTODO}

Se realizó un estudio retrospectivo, donde se incluyeron a todos los pacientes con fisura de labio y/o paladar atendidos bajo tratamiento de ortopedia prequirúrgica en el Consultorio Miraflores, Región de la Araucanía, entre los años 2003 y 2012, y que hayan sido dados de alta para ser sometidos a una operación primaria. En todos los casos, las fisuras fueron categorizadas según la clasificación de Kernahan (Fig. 1).

La muestra se constituyó por 100 pacientes de ambos sexos. Este estudio fue aprobado por el comité de ética de Servicio de Salud Araucanía Sur y autorizado por el Director del Consultorio Miraflores. 


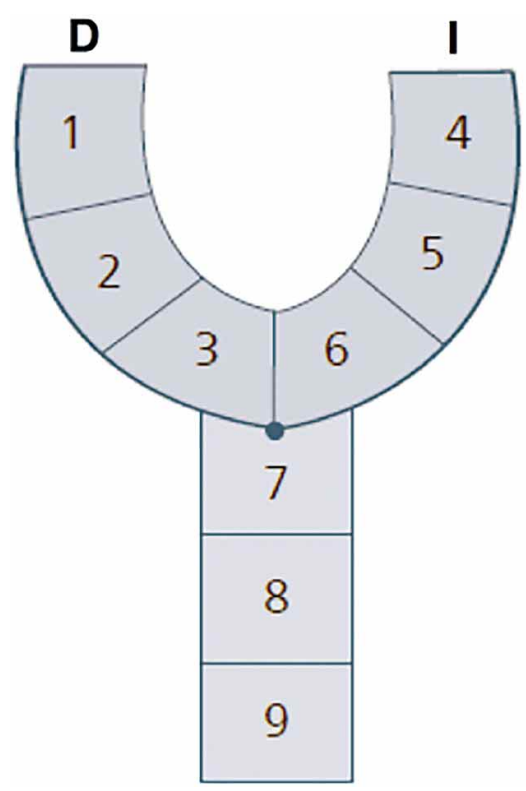

Fig. 1. Clasificación tipo "Y" de Kernahan para fisuras labio-palatinas. Las áreas 1 y 4 representan los lados derecho e izquierdo de los labios, respectivamente. El alvéolo está representado por las áreas 2 y 5 , el paladar duro anterior al foramen incisivo por las áreas 3 y 6 , el paladar duro posterior al foramen incisivo por las áreas 7 y 8 , y el velo del paladar por el área 9.

Se recurrió a la recolección y análisis de datos entregados por las fichas clínicas de los pacientes bajo tratamiento de ortopedia prequirúrgica. No hubo intervención directa sobre pacientes.

La información recolectada fue traspasasa al programa Excel para categorizar y clasificar a los pacientes. El posterior análisis estadístico se realizó con el programa STATA12.

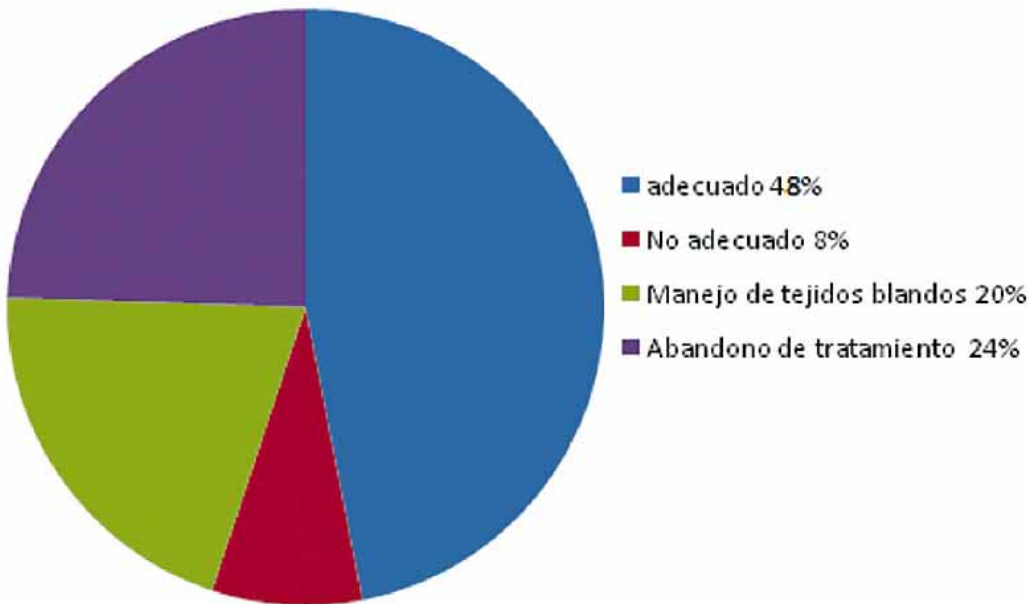

Fig. 2. Gráfica que muestra porcentualmente el resultado del tratamiento ortopédico de todos los pacientes con fisuras labio-palatinas atendidos en el Consultorio Miraflores entre los años 2003 y 2012.

\section{RESULTADOS}

Respecto al acercamiento de los procesos alveolares en los pacientes atendidos, el $48 \%$ logró un adecuado avance de los procesos alveolares, un $20 \%$ recibió el tratamiento pero enfocado en el manejo de tejidos blandos de la cara, un $8 \%$ no logro un acercamiento adecuado y un $24 \%$ de los pacientes abandono el tratamiento (Fig. 2).

En relación al acercamiento de los procesos alveolares y las mediciones de las fisuras, se pudo encontrar que el promedio de la medición inicial del lado derecho fue de $7,6 \mathrm{~mm}$, y del lado izquierdo $8,79 \mathrm{~mm}$. El promedio de la medición final del lado derecho fue de $2,16 \mathrm{~mm}$, donde en 21 pacientes fue exitoso, correspondiente al $84 \%$ de los casos del lado derecho. El promedio de la medición final del lado izquierdo fue de 3,08 $\mathrm{mm}$, donde en 29 de ellos fue exitoso $70,7 \%$ de los casos del lado izquierdo.

Al evaluar la edad de inicio de tratamiento vs. acercamiento de los procesos alveolares, no existen diferencias significativas entre la edad de inicio al tratamiento la adecuación a los procesos alveolares $(p=0,327)$ (Tabla I).

En relación al número total de atenciones vs. acercamiento de los procesos alveolares, se observan diferencias significativas $(p=0,0,038)$

Tabla I. Edad de inicio de tratamiento de ortopedia prequirúrgica vs. acercamiento de procesos alveolares de los pacientes atendidos entre los años 2003 y 2012. Prueba de ANOVA.

\begin{tabular}{lccccr}
\hline & Suma de cuadrados & GI & Media cuadrática & F & Sig. \\
\hline Inter-grupos & 606,289 & 3 & 202,096 & 1,167 & 0,327 \\
Intra-grupos & 16456,398 & 95 & 173,225 & & \\
Total & 17062,687 & 98 & & & \\
\hline
\end{tabular}


(Tabla II). Se comprobó que las mayores diferencias se presentaron entre un adecuado acercamiento de los procesos y el abandono del tratamiento.

Al correlacionar el tiempo total de tratamiento vs. acercamiento de los procesos alveolares, se ob- servaron diferencias significativas $(p=0,022)$ (Tabla II). Las diferencias estaban presentes en el adecuado acercamiento de los procesos y el manejo de tejidos blandos $(p=0,014)$, así como en el acercamiento no adecuado y el manejo de tejidos blandos $(p=0,017)$.

Tabla II. Número total de atenciones ortopédicsa prequirúrgicas vs. acercamiento de los procesos alveolares de los pacientes atendidos entre los años 2003 y 2012. Prueba de ANOVA.

\begin{tabular}{lccccc}
\hline & Suma de cuadrados & GI & Media cuadrática & F & Sig. \\
\hline Inter-grupos & 387,024 & 3 & 129,008 & 2,933 & 0,038 \\
Intra-grupos & 4091,017 & 93 & 43,989 & & \\
Total & 4478,041 & 96 & & &
\end{tabular}

Tabla III. Tiempo total de tratamiento de ortopedia prequirúrgica vs. acercamiento de procesos alveolares de los pacientes atendidos entre los años 2003 y 2012. Prueba de ANOVA.

\begin{tabular}{lccccc}
\hline & Suma de cuadrados & Gl & Media cuadrática & F & Sig. \\
\hline Inter-grupos & 20277,347 & 3 & 6759,116 & 3,360 & 0,022 \\
Intra-grupos & 183085,874 & 91 & 2011,933 & & \\
Total & 203363,221 & 94 & & & \\
\hline
\end{tabular}

\section{DISCUSIÓN}

Las fisuras de labio y paladar son malformaciones comunes en nuestra realidad, pudiendo encontrárse en conjunto con otras malformaciones congénitas. Estas fisuras acarrean un amplio número de desórdenes asociados, como desordenes de alimentación, problemas de lenguaje, deficiencias dentales y maloclusiones entre otras. Existen además consecuencias estéticas de las malformaciones faciales producidas, que pudiesen crear problemas mentales y psicológicos no solo en los individuos afectados, sino como también en sus familiares. En este estudio, un $46 \%$ de los pacientes atendidos logró un adecuado acercamiento de los procesos alveolares mediante la técnica prequirúrgica de Grayson, lo que representa un mejoría respecto a lo reportado por Iturriaga et al. (2010), quienes ya habían estudiado a los pacientes atendidos en la región de la Araucanía bajo el mismo tratamiento, en el periodo 2003-2009, en donde bajo parámetros similares, se obtuvo que un buen resultado fue se logró en el $36 \%$ del total. Poder determinar la cantidad de paciente que logro un acercamiento adecuado de los procesos alveolares mediante la técnica prequirúrgica de Grayson, permite al clínico no solo dimensionar el efecto del tratamiento llevado a cabo en los pacien- tes, sino que también ayuda a pesquisar los problemas que pudiesen estarse presentando y que impiden un mejor resultado. Es una herramienta útil para enseñar a los padres y/o tutores lo logrado con la técnica, mejorando así la confianza en el tratamiento, con ello, la adhesión al mismo y últimamente logrando un resultado óptimo en los pacientes.

La información indica que la mayoría de los pacientes logró un adecuado acercamiento de los procesos alveolares mediante la técnica aplicada, lo que demuestra que el tratamiento funciona y que, por ende, es apropiado para la preparación de pacientes fisurados a cirugía primaria. Los beneficios perseguidos con este tratamiento, en la última década, mediante las distintas técnicas, son descritos por la Guía clínica de Fisura Labiopalatina del MINSAL como: 1). Buena estética nasal de largo plazo (corregir la malposición de los cartílagos nasales y la base alar en el lado afectado, alargar la columela, etc.).; 2). Menor número de procedimientos quirúrgicos en la nariz; 3). Acercar los segmentos maxilares, y modelar los tejidos blandos para disminuir la tensión de las partes blandas, y favorecer la reparación del labio y nariz; 4). Obtener una platafor- 
ma maxilar estable; 5). Si se incluye la gingivoperiosteoplastía en el protocolo, se espera reducir la necesidad de injertos óseos en la mayoría de los pacientes; 6). Crecimiento sin mayores alteraciones en comparación a los pacientes con tratamiento tradicional, y 7). Ahorro para el paciente y la sociedad debido a la reducción en los procedimientos quirúrgicos.

En los análisis realizados, no se encontró información con la cual corroborar información respecto la edad de inicio del tratamiento y el resultado final de este, se ha indicado por Muñoz \& Castro (2006), que el inicio del tratamiento debiese ser inmediato luego del nacimiento. Sin embargo el análisis estadístico indicó que la edad no tendría relación con el resultado final del tratamiento, pudiendo atribuírselo a que el rango de edad es corto, solo se presentan días de diferencia, las que estadísticamente no serian significativos en el resultado final obtenido.

En lo que respecta a la edad en la que fueron dados de alta los pacientes, se registró un promedio de 130 días, bastante cercano al tiempo que se indica según Grayson \& Shetye, que podría variar entre los 3 a 4 meses de edad, pudiendo aumentar en caso de atender a pacientes con fisuras bilaterales hasta los 6 meses. Este periódo deber ser tomado en consideración, ya que en relación al número total de atenciones, éstas incidieron negativamente en el acercamiento de los pro- cesos alveolares, principalmente por el abandono del tratamiento, confirmando que con un mayor número de controles, existe una mayor probabilidad de lograr un adecuado acercamiento de los procesos alveolares con la técnica.

En conclusión, el conocimiento sobre el tratamiento realizado, su importancia y el soporte del mismo de de vital relevancia para la mejoría de los pacientes. Se requiere de un apoyo familiar cercano, atento y que trabaje en conjunto con los profesionales, con tal de generar un equipo de trabajo en torno al paciente llevando a cabo las tareas solicitadas y controlando la mantención del tratamiento estipulado. Es este rol protagónico que tiene el soporte familiar el que debe ser reforzado, en el que nos enfocamos con información clara y precisa, permitiendo un rápido entendimiento de la situación y generando un conocimiento acorde a las necesidades del paciente, es esa la importancia que radica en la construcción del manual de promoción, educación y cuidados especiales de pacientes bajo el tratamiento ya mencionado.

Se debe ampliar la investigación con los pacientes fisurados, existen pocos datos a nivel nacional sobre el área, se presenta como un campo amplio en el cual desarrollarse y que requiere un mayor soporte profesional para mejorar la atención y con ello la calidad de vida de los pacientes.

FUENTES, J; SILVA, M.; CANTÍN, M. \& LLERMALY, S. Approaching of the alveolar processes using presurgical orthopedics in patients with cleft lip and palate. Int. J. Odontostomat., 8(1):119-124, 2014.

ABSTRACT: Oral clefts are a heterogeneous group of congenital malformations of multifactorial origin. Cleft lip and palate is one of the most frequent alterations of the fusion of the labial medial, lateral and palatal processes during embryonic development with Chile having a higher prevalence than the rest of the world. Treatment includes various procedures such as preoperative techniques. The objective of this study was to evaluate whether preoperative treatment prepares patients with cleft lip and/or cleft palate for primary surgery. We performed a retrospective study involving 100 male and female patients with cleft lip and/or palate admitted for presurgical orthopedic treatment at the Miraflores primary healthcare service, Temuco, Chile, between 2003 and 2012. Clefts were categorized according to Kernahan classification. Of the treatments, 46\% achieved a suitable approach for surgery and $20 \%$ required only soft tissue management. Additionally, $8 \%$ percent did not achieve an adequate approach and twenty-four percent of patients discontinued therapy. No differences were observed between age at onset of treatment vs. alveolar process approach. Soft tissue management was significantly correlated with the total treatment time and the approach of the alveolar processes. To determine the number of patients achieving an adequate approach to the alveolar process by preoperative technique, allows the clinician to gauge the effect of treatment, find and prevent problems thereby obtaining better results.

KEY WORDS: cleft lip and palate, preoperative stage, Grayson stage.

\section{REFERENCIAS BIBLIOGRÁFICAS}

Bermudez, L.; Lizarraga, A. K. \& Carter, V. How and why clefts occur? Norfolk, Operation Smile, Research and Outcomes Department, 2009.
Bille, C.; Skytthe, A.; Vach, W.; Knudsen, L. B.; Andersen, A. M.; Murray, J. C. \& Christensen, K. Parent's age and the risk of oral clefts. Epidemiology, 16(3):311-6, 2005. 
FUENTES, J; SILVA, M.; CANTíN, M. \& LLERMALY, S. Acercamiento de los procesos alveolares mediante ortopedia prequirúrgica en pacientes con labio y paladar fisurado. Int. J. Odontostomat., 8(1):119-124, 2014.

Ford, A.; Tastets, M. \& Caceres, A. Tratamiento de la fisura labio palatina. Rev. Med. Clin. Condes, 21(1):16-25, 2010.

Grayson, B. H. \& Shetye, P. R. Presurgical nasoalveolar moulding treatment in cleft lip and palate patients. Indian J. Plast. Surg., 42(Suppl.):S56-61, 2009.

Iturriaga, P.; Almeida, E.; Montecinos, G. \& Suárez, V. Tratamiento ortopédico prequirúrgico en fisura labio palatina, implementación de la técnica y seguimiento a corto plazo. Rev. Ped. Elec., 7(3):59, 2010.

Little, J.; Cardy, A. \& Munger, R. G. Tobacco smoking and oral clefts: a meta-analysis. Bull. World Health Org., 82(3):213-8, 2004.

Ministerio de Salud. Guía Clínica Fisura Labiopalatina. Santiago, MINSAL, 2009. Disponible en: http:// www.redsalud.gov.cl/archivos/guiasges/ FisuraLabiopalatinaR_Mayo10.pdf

Muñoz, P. A. \& Castro, L. L. Ortopedia tridimensional y manejo preoperatorio de tejidos blandos en labio y paladar hendidos. Cir. Plast., 16(1):6-12, 2006.

Nazer, J. Malformaciones Congénitas. Santiago, Edición Servicio Neonatología Hospital Clínico Universidad de Chile, 2011, pp.218-23. Cap. 30.

Romitti, P. A.; Herring, A. M.; Dennis, L. K. \& WongGibbons, D. L. Meta-analysis: pesticides and orofacial clefts. Cleft Palate Craniofac. J., 44(4):358-65, 2007.

Rossell, P. Manejo Prequirúrgico. En: Rossell, P. Tratamiento de la Fisura Labio Palatina. Lima, Fondo Editorial de la UNMSM, 2009. pp.57-67, 117-34.

Wyszynski, D. F.; Duffy, D. L. \& Beaty, T. H. Maternal cigarette smoking and oral clefts: a meta-analysis. Cleft Palate Craniofac. J., 34(3):206-10, 1997.
Dirección para Correspondencia:

Dr. Jorge Fuentes Nawrath

Depto. Odontología Integral

Manuel Montt 110, Temuco

CHILE

Email: jorge.fuentes@ufrontera.cl

Recibido : 05-10-2013

Aceptado: 23-12-2013 